\title{
Integrating Automated Fingerprint-based Attendance into a University Portal System
}

\author{
Joke A. Badejo, Chinonso C. Eke, Segun I. Popoola, Tiwalade O. Odu, Aderemi \\ A. Atayero
}

Department of Electrical and Information Engineering Covenant

University Canaanland-Ota, Nigeria

joke.badejo@covenantuniversity.edu.ng,chinonsoeke@gmail.com, segun.popoola@stu.cu.edu.ng, tiwalade.odu@cu.edu.ng, atayero@covenantuniversity.edu.ng

\begin{abstract}
Student learning and academic performance hinge largely on frequency of class attendance and participation. The fingerprint recognition system aims at providing an accurate and efficient attendance management service to staff and students within an existing portal system. The integration of a unique and accurate identification system into the existing portal system offers at least, two advantages: accurate and efficient analysis and reporting of student attendance on a continuous basis; and also facilitating the provision of personalized services, enhancing user experience altogether. An integrated portal system was developed to automate attendance management and tested for fifty students in five attempts. The $98 \%$ accuracy achieved by the system points to the feasibility of large scale deployment and interoperability of multiple devices using existing technology infrastructure.
\end{abstract}

Keywords - fingerprint recognition; biometrics; attendance system; education portal system; learning analytics. 